\title{
Design of Velocity PID-Fuzzy Power System Stabilizer Using Particle Swarm Optimization
}

\author{
T. HUSSEIN ELMENFY \\ Electrical and Electronics Engineering Dept, University of Benghazi, Benghazi, LIBYA \\ Tawfiq.elmenfy@uob.edu.ly
}

\begin{abstract}
Power system stabilizer (PSS) is used to damping power system local and interarea modes of oscillation. It will act as additional signal through the generator excitation system which produces a component of electrical torque in phase with speed variation. Fuzzy controller is nonlinear. It is more difficult to set the fuzzy controller gains compared to conventional PID controller. This research proposed velocity (PID-FPSS) its gains are tuned off-line by particle swarm optimization techniques (PSO). The objectives are to damp local-area mode of oscillations that occur following power system disturbances. The effectiveness of the proposed technique is illustrated by applying the velocity PID-FPSS to a single-machine infinite bus power system that is typically used in the literature to test the performance of power system stabilizers. The simulation studies has been tested in MATLAB SIMULINK package. A comparison between the proposed PID-FPSS and a well-tuned and designed robust power system stabilizer (RPSS) confirms the superiority of the PID-FPSS.
\end{abstract}

Keywords: Velocity PID Controller, Fuzzy logic system, , Swarm optimization technique.

Received: October 30, 2020. Revised: January 10, 2021. Accepted: February 19, 2021. Published: February $28,2021$.

\section{Introduction}

Power system stabilizers are used for many years as supplementary control signal in automatic voltage regulator (AVR) to add damping of the electromechanical oscillations. It will act through the generator excitation system which produces a component of electrical torque in phase with speed variations to the speed deviation $[1,2]$.

The fixed parameters of conventional PSS (CPSS) [3] are derived from mathematical linear model of the plant and designed at one operating condition. This confirm that CPSS is not a suitable for a wide range of operating conditions. In CPSS the parameters are evaluated at particular loading conditions.

The design requirements are considered in [4] introduced both time domain and frequency domain specifications which are initially specified before designing the PSS controller, the optimization based linear control design technique is used to determine the optimal PSS parameters. [5] introduces fuzzy expert system, the generator speed deviation and acceleration with scaling factors are chosen as input signals to fuzzy logic power system stabilizer and the desired output is integral square time square error and simulation results shows the superior of proposed technique over CPSS..

Fuzzy logic-based PSS for stability enhancement of a two-area four machine system are designed in [6]. In order to accomplish the stability enhancement, speed deviation $(\Delta \omega)$ and active power deviation $(\Delta P)$ of the rotor synchronous generator were taken as the inputs to the fuzzy logic controller. These signals take significant effects on damping the electromechanical oscillations. The stability signals were processed using fuzzy membership function depending on the variables. The design of a proportional, derivative and integral (PID) based power system stabilizer (PSS) introduced in [7], that design carried out using a new Meta heuristic harmony search algorithm (HSA) to optimize the parameters. The design of PID controller is considered with an objective function based on eigenvalue shifting to guarantee the stability of nonlinear plant for a wide range of conditions using HSA. The use of the cost function to minimize the summation of the absolute value is used to design Conventional PSS in [8], and the absolute value is composed of the difference between the square of required compensation phase in excitation system and the square of phase provided by CPSS which should be minimum.

In this paper, a velocity PID-Fuzzy Power System Stabilizer and its gains are tuned by Particle Swarm Optimization is presented. This stabilizer uses the speed deviation, the derivative speed deviation and the output power as inputs to the fuzzy controller (obtained online and assumed to be measured from the output of the plant). This controller is used to provide the necessary damping to the plant. 


\section{Velocity PID Controller}

On form of the controller widely used in the industrial process control is called a three terms of ProportionalIntegral-Derivative (PID) controller [9]. The PID controller are the most commonly used in industrial process control. The PID controller has the following formula.

$C(t)=c_{0+} K_{p}\left[e(t)+\frac{1}{T_{i}} \int_{0}^{t} e(t) d t+T_{d} \frac{d}{d t} e(t)\right] \ldots .(1)$

Where:

$K_{p}$ : Proportional gain

$T_{i}$ : Integration time

$T_{d}:$ Derivative time

$c_{0}:$ Controller bias

$e(t)=y_{s p}-y_{s}=$ set point- process measurement.

The disadvantage of the controller in eq.(1), the derivative of the error is subject to the derivative kick and the integral part not compatible to the digital computer.

Also set-point can change instantly in a step change- this cause $e(t)$ to have a step change whenever set-point is changed. The sudden step change in $e(t)$ cause the derivative of $e(t)$ to be infinite- this cause the derivative term to be huge whenever the set-point is changed (derivative kick).

To eliminate the derivative kick, the derivative part should be calculated based on the measurement $\left(y_{s}\right)$ rather than the error $\mathrm{e}(\mathrm{t})$ as in eq.(2).

$C(t)=c_{0}+K_{p}\left[e(t)+\frac{1}{T_{i}} \int_{0}^{t} e(t) d t+T_{d} \frac{d}{d t} y_{s}(t)\right]$

The integral part not compatible with digital computers.

Since, $e(t)=y_{s p}-y_{s}$

Then,

$\frac{d}{d t} e(t)=\frac{d}{d t} y_{s p}-\frac{d}{d t} y_{s}$

Under normal operating conditions, when setpoint is not changing: $\frac{d}{d t} e(t)=-\frac{d}{d t} y_{s}$

The integral part can be replaced with summation and the derivative part replaced by finite difference.

Then eq.(2) can be rewrite at time (t) as:

$$
\begin{aligned}
C(t)=c_{0}+K_{p}[e(t) & \\
& +\frac{\Delta t}{T_{i}} \sum_{i=1}^{n} e(\Delta t) \\
& \left.-T_{d}\left(\frac{y_{s}(t)-y_{s}(t-\Delta t)}{\Delta t}\right)\right] \ldots
\end{aligned}
$$

In eq.(6) summation can cause overflow errors on digital computers and $(\Delta t)$ must be small compared to process response time.

Velocity PID controller form development as:

- Evaluate position for current state form at (t) to get $\mathrm{c}(\mathrm{t})$.

- Evaluate position for the past state form at $(t-$ $\Delta t)$ to get $c(t-\Delta t)$.

- $\quad$ Subtract to get $\Delta c(t)=c(t)-c(t-\Delta t)$

$C(t-\Delta t)=c_{0}+K_{p}\left[e(t-\Delta t)+\frac{\Delta t}{T_{i}} \sum_{i=1}^{n} e(\Delta t)-\right.$

$\left.T_{d}\left(\frac{y_{S}(t-\Delta t)-y_{S}(t-2 \Delta t)}{\Delta t}\right)\right]$

Subtract eq.(6) from eq. (7) to get velocity form:

$\Delta C(t)=K_{p}\left[e(t)-e(t-\Delta t)+\frac{\Delta t}{T_{i}} e(t)-\right.$

$\left.T_{d}\left(\frac{y_{S}(t)-2 y_{S}(t-\Delta t)+y_{S}(t-2 \Delta t)}{\Delta t}\right)\right]$

The gains to be tuned in velocity PID controller are $K_{p}$, $T_{i}$ and $T_{d}$.

\section{Overview of Particle Swarm Optimization}

Particle swarm optimization (PSO) is a population based algorithm and it is collection of particles move in steps of regions, developed by Dr. Eberhart and Dr. Kennedy in 1995, explored by social behavior of bird flocking or fish schooling [10].

The features of the PSO are as follows

- The PSO technique is based on searching on groups or swarm such as fish schooling and bird flocking. 
- $\quad$ PSO is easy to implement as algorithm which is written in a very few lines of code and there are few parameters to adjust.

- $\quad$ PSO learned from the experience and used it to solve the optimization problems

PSO is a method used for optimization of continuous nonlinear functions. According to the research results for the bird flocking, birds find the foods by flocking not by each individual. Each particle keeps track of its coordinates in the space, which are associated with the best solution. This value is called pbest. Second best value that is tracked by the global version of the particle swarm optimizer is the overall best value, and its location obtained so far by any particle in the, is called gbest.

The PSO concept is, at each iteration, changing the velocity and the postion of each particle toward its pbest and gbest location.

The modified velocity of each agent can be calculated using the current velocity and his position from pbest and gbest as shown below

$$
\begin{array}{r}
v_{i}^{k+1}=w_{i} v_{i}^{k}+c_{1} \text { rand } \times\left(\text { pbest }-s_{i}^{k}\right)+c_{2} \text { rand } \\
\times\left(\text { gbest }-s_{i}^{k}\right) \ldots \ldots \ldots \ldots(9)
\end{array}
$$

Where,

$v_{i}^{k} \quad$ : current velocity of particle $\mathrm{i}$ at iteration $\mathrm{k}$,

$v_{i}^{k+1}:$ modified velocity of particle $\mathrm{i}$,

rand : random number between 0 and 1 ,

$s_{i}^{k} \quad$ : current position of particle $\mathrm{i}$ at iteration $\mathrm{k}$,

pbest : pbest of particle $\mathrm{i}$,

gbest : gbest of particle i,

$w_{i} \quad$ : weight function for velocity of agent $\mathrm{i}$,

$c_{i} \quad$ : weight coefficient.

Using the eq. (9), a appropriate velocity that gradually gets close to pbest and gbest can be calculated. The current position at iteration I can be modified by the following equation.

$$
s_{i}^{k+1}=s_{i}^{k}+v_{i}^{k+1}
$$

Fig. 3 shows the concepts of modification of a searching point by PSO.

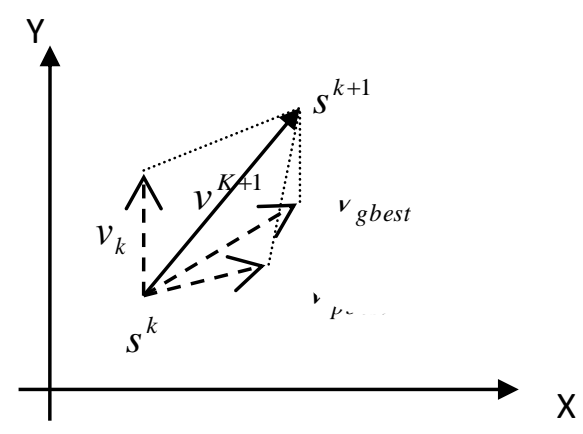

Fig. (1): Concept of modification of a searching point

$s^{k} \quad$ : current searching point,

$s^{k+1}$ : modified searching point,

$v^{k}$ : current velocity,

$v^{k+1}:$ modified velocity,

$v_{\text {pbest }}$ : velocity based on pbest,

$v_{\text {gbest }}$ : velocity based on gbest.

Fig. (2) shows a searching concept with agent in a solution space.

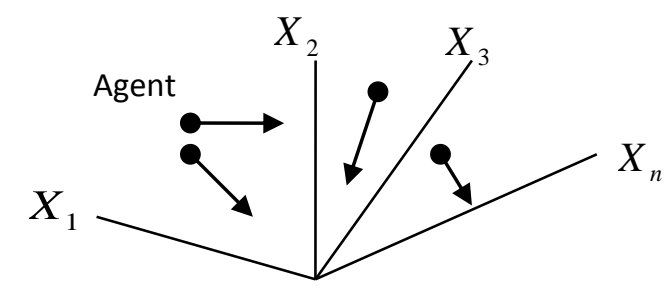

Fig. (2): Searching concept with agent in a solution space by PSO

\section{The algorithm of PSO}

The proposed algorithm of PSO for searching the optimal values of the elements $k$ as follow

1. Initialize the swarm with random positions and velocities.

2. Calculate the fitness function of each particle by

$0.5 \times \operatorname{sum}\left(\omega-\omega_{d}\right)^{2} \times t$.

Where,

$$
\begin{aligned}
& \omega: \text { actual speed } \\
& \omega_{d}: \text { desired speed }
\end{aligned}
$$

3. Determine the pbest and the gbest positions.

4. Update the velocity of particle using Eq. (9).

5. Update the position of particle using Eq. (10).

6. If the evaluation value of each particle is better than the previous pbest, the value is set to gbest. If the best pbest (local version) is better than gbest (global version), then the next value is set to gbest. 
7. if the iteration number reaches the maximum iteration number, then go to step 8. Otherwise, go to step 2 .

plot pbest, gbest and compute the Eq. (11) for the control candidates with optimal values of $k$.

\section{Design of Velocity PID-FPSS}

The control problem is to design velocity PID controller in eq. (8) as fuzzy logic controller. Velocity PID controller can be converted into a fuzzy controller in a nonlinear manner to enhance robustness.

Consider fuzzy PID controller with inputs as $\left(e, P_{e}\right)$ and output $(u)$.

Where:

e: speed deviation.

$P_{e}:$ electrical power.

u: control signal.

The proposed PID-FPSS is designed as follow [11]:

1. Identification of inputs and output variables as $\left(e, P_{e} \& u\right)$.

2. Construction the fuzzy control rules as in table (1).

3. Selection of the compositional rule of inference.

4. Defuzzification method, transformation of fuzzy control set into fuzzy control action.

The center of area method (COA) is used for defuzzification. Gaussian membership function to represent the inputs/ output linguistic variables. For each inputs/ output variables, three labels are defined as shown in Fig. (3).

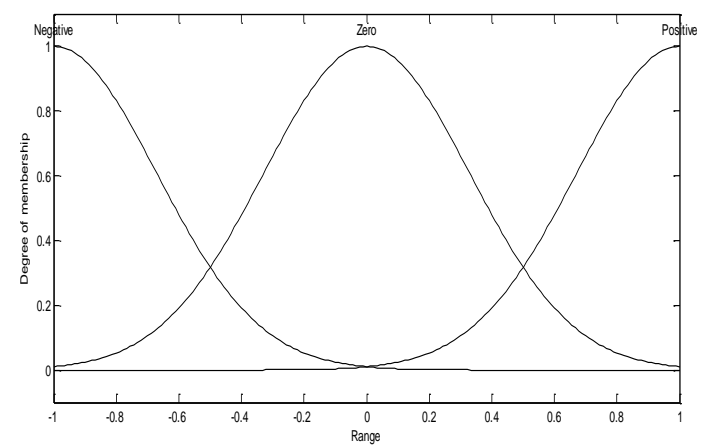

Fig. (3): Fuzzy variable, Xi, three membership function

\begin{tabular}{||l||c||c||c||}
\hline \multicolumn{1}{|c||}{$\Delta \dot{\omega}$} & $N$ & $Z$ & $P$ \\
\hline \hline$N$ & $N$ & $N$ & $Z$ \\
\hline \hline$Z$ & $N$ & $Z$ & $P$ \\
\hline$P$ & $Z$ & $P$ & $P$ \\
\hline
\end{tabular}

Table 1: Fuzzy-logic PSS rules

$\mathrm{N}, \mathrm{Z}$ and $\mathrm{P}$ stand for negative, zero and positive. The value $x_{\max }$ and $x_{\min }$ represent maximum and minimum variation of the input and output signals. The values are selected based on simulation information. A decision table is constructed consisting of 9 rules. An example of the ith rule is:

\section{If $\Delta \omega$ is $\mathbf{N}$ and $\Delta \omega$ is $\mathbf{P}$ then $\mathrm{U}$ is $\mathrm{Z}$}

A symmetrical fuzzy rule set is used to describe the PID-FPSS behavior as shown in table 1 .

The designing procedure of a FLC are explained in details in [1].

The PID-FPSS parameters are getting by particle swarm optimization (PSO) are:

$K_{p}=7 \quad ; T_{i}=0.32 \quad ; T_{d}=0.12$

\section{Implementation to Single Machine Infinite-Bus Model}

Fig. (4) shows the system under study. It comprises a single machine connected to an infinite bus through a tie line. The machine is equipped with a static exciter. The system is represented by the block diagram proposed by deMello and Concordia [12]. The generator is represented by third-order nonlinear model and the static exciter is represented by a first-order model. The nonlinear equations of the machine are given in [13].

\section{Simulation Results}

The PSS that is used for comparison is a simple robust power system stabilizer (RPSS) is designed in [13] that can properly function over a wide range of operating conditions and extend the machine load ability. The lead compensator design is achieved by drawing the root loci for a finite number of extreme characteristics polynomials. Such polynomials are obtained, using the 
kharitonov theorem [13], to reflect wide loading conditions on characteristics equation coefficient.

For a comparison purpose, the system is configured to switch between two control techniques. In order to show the response of PID-FPSS and RPSS.

To investigate the power system performance. The system responses to a three-phase fault At $t=0 \mathrm{sec}$. and cleared after $100 \mathrm{~ms}$, will show the response of the system for two operating conditions.

The performance of each control law was followed by the performance index:

$J_{P}=\sum \Delta \omega^{2}$

The selected loading conditions are as follows:

Operating Condition 1: Electrical Power $\left(P_{e}=0.8 p u\right)$.

Operating Condition 2: Electrical Power $\left(P_{e}=0.3 p u\right)$

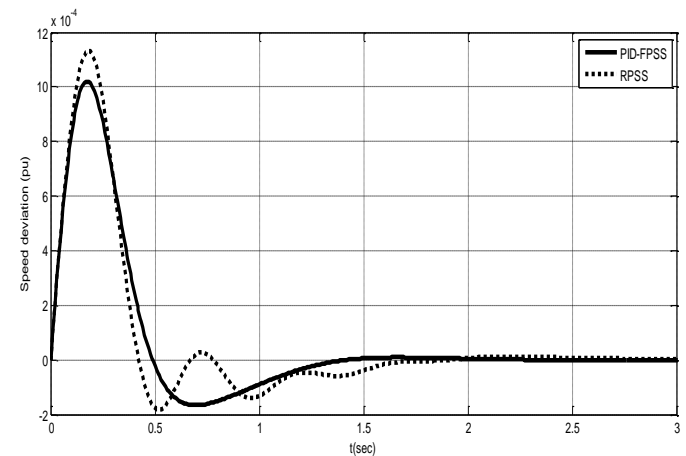

Fig. (4): speed deviation response at $\left(P_{e}=0.8 p u\right)$

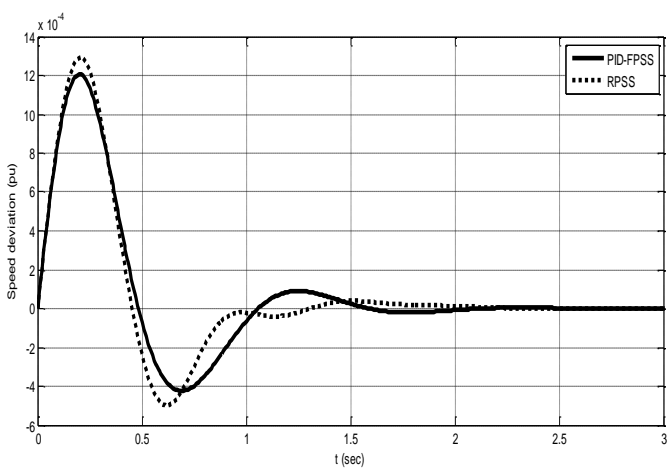

Fig. (5): speed deviation response at $\left(P_{e}=0.3 p u\right)$

TABLE (2). The performance index, at operating condition 1

\begin{tabular}{|l|c||}
\hline \hline Controller & Speed \\
\hline PID-Fuzzy PSS & 225 \\
& \\
\hline
\end{tabular}

\begin{tabular}{|l|l|}
\hline Robust PSS & 255 \\
\hline
\end{tabular}

TABLE (3). The performance index, at operating condition 2

\begin{tabular}{|c|c|}
\hline Controller & Speed Deviation \\
\hline PID-Fuzzy PSS & 450 \\
\hline Robust PSS & 501 \\
\hline
\end{tabular}

\section{Conclusion}

PID-FPSS technique was designed based velocity PID controller and its gains are tunes by particle swarm optimization technique.

Power system stabilizer. It was developed based on linguistic rules of fuzzy basis functions. The performance of the proposed stabilizer was investigated and compared with the robust PSS through dynamic simulations of a single machine infinite bus power system. The obtained simulations have shown that a significant improvement can be achieved with PID-FPSS design for a different operating points.

\section{References}

[1].W. Watson and Manchur, "Experience with supplementary damping signals for generator excitation systems" IEEE Trans. on power apparatus and systems, vol PAS-1973, PP. 193-203.

[2]. F. P. deMello, L. N. Hannett and M. Undrill " Practical approaches to supplementary stabilizing from accelerating power" IEEE Trans. On power apparatus and systems, vol PAS1978, PP. 1515-1522.

[3].H. Joe Chow, E. G. Boukarim and A. Murdoch " Power system stabilizer as undergraduate control design project" IEEE Trans. On power apparatus and systems, vol PAS-2004, PP. 144-151. 
[4].G. Y. Rajaa Vikhram, S. Latha " Design of power system stabilizer for power system damping improvement with multiple design requirements" international journal of soft computing and Engineering (IJSCE) ISSN: 223123077, vol. 2, 2012.

[5].M. Megala, Rajan, C. Cristober Asir " Design of fuzzy logic power system stabilizer in multi-machine power system" proceedings of the international conference on frontiers of intelligent computing: theory and application 2013.

[6].P. Kripakaran, M. Vigneshan, S. Asvnth, B. Sivachandiran " performance of power system stabilizer using fuzzy logic controller" journal of electrical and electronic engineering (JOSR-JEEE), vol. 9. 2014, pp. 42-49.

[7]. D. K. Sambariya, R. Prasad " Design of robust PID power system stabilizer for multimachine power system using HS algorithm" American Journal of Electrical and Electronic Engineering vol. 3, No. 3, 2015, pp 75-82.

[8].X. Li, Z. Wang, Junhua Xu, and B. Chen "Power system stabilizer parameters designing based on genetic simulated annealing algorithm" JOCET 2016 vol.4, No.3, 2016, pp 178-182.

[9].Haider A. and Sulochana W. "Intelligent PID Controller Tuning for Higher Order Process System" International Journal of $\mathrm{u}-$ and e- Service, Science and Technology Vol.8, No. 6, 2015, pp.323330.

[10]. T. Hussein and A. Shamekh " Performance Assessment of Fuzzy Logic Power System Stabilizer on North Benghazi Power Plant" Hindawi
Publishing Corporationn Conference Papers in Engineering, 2013.

[11]. John H. " Fuzzy control and identification" puplished by John Wiley \& Sons, 2010.

[12]. F. P. Demello, C. Concordia " Concepts of Synchronous Machine Stability as Affected Excitation Control" IEEE Transactions on Power Apparatus and Systems, vol. pas-88, no. , pp 316328. 1969.

[13]. H. M. Soliman, A. L. Elshafei, A. A. Shaltout and M. F. Morsi " Robust Power System Stabilizer" IEE Proceeding on Electrical Power Aplication, vol 14, pp 285-291, 2000.

\section{Creative Commons Attribution License 4.0 (Attribution 4.0 International, CC BY 4.0)}

This article is published under the terms of the Creative Commons Attribution License 4.0

https://creativecommons.org/licenses/by/4.0/deed.en US 